\title{
Apoptosis and cell cycle arrest of human colorectal cancer cell line HT-29 induced by vanillin.
}

\begin{abstract}
Background: Vanillin is responsible for the flavor and smell of vanilla, a widely used flavoring agent. Previous studies showed that vanillin could enhance the repair of mutations and thus function as an anti-mutagen. However, its role in cancer, a disease that is closely related to mutation has not yet been fully elucidated. Methods: Hence, this study investigated the cytolytic and cytostatic properties of vanillin against HT-29, a human colorectal cancer cell line. Methods used including cell viability assay, acridine orange (AO)-ethidium bromide (EB) double staining cell morphological analysis, Cell cycle analysis, annexin Vpropidium iodide apoptosis test and 5-bromo-2-deoxyuridine (BrdU)-labeling cell proliferation assay. Results: Results showed that apoptosis was induced by vanillin and the IC50 for HT-29 and NIH/3T3 normal cell lines were $400 \mu \mathrm{g} / \mathrm{ml}$ and $1000 \mu \mathrm{g} / \mathrm{ml}$, respectively. Different concentrations of vanillin arrest cell cycle at different checkpoints. 5-Bromo-2deoxyuridine-labeling cell proliferation assay showed that G0/G1 arrest was achieved at lower concentration of vanillin $(200 \mu \mathrm{g} / \mathrm{ml})$ while cell cycle analysis by flow cytometer showed that $\mathrm{G} 2 / \mathrm{M}$ arrest occurs at higher concentration of vanillin $(1000 \mu \mathrm{g} / \mathrm{ml})$. Conclusion: Cytolytic and cytostatic effects shown by vanillin showed that it could be a useful colorectal cancer preventive agent. Further in vivo study should be carried out to confirm that similar effects could happen in animals.
\end{abstract}

Keyword: Cell viability; MTS assay; Cell morphology; Acridine orange-ethidium bromide; Cell cycle; Apoptosis; Annexin V-propidium iodide; 5-Bromo-2-deoxyuridine (BrdU); Anti-proliferation 